\title{
FACTORES QUE EXPLICAN EL CAMBIO DE LA IMAGEN DE UN DESTINO TURÍSTICO ANTES Y DESPUÉS DE LA VISITA
}

\author{
Asunción Beerli Palacio* \\ Josefa D. Martín Santana* \\ Universidad de Las Palmas de Gran Canaria \\ Patrizio A. Nazzareno** \\ Nazzareno Consulting S.L.U.
}

\section{RESUMEN}

El presente trabajo ha tenido como principal objetivo estudiar y analizar empíricamente los factores que explican el cambio de imagen antes y después de la visita a un destino turístico. En el estudio empírico se ha utilizado una muestra representativa de turistas de la isla de Tenerife (Islas Canarias), que es uno de los principales destinos turísticos de España. Los resultados ponen de manifiesto que los factores que mejor explican el cambio de imagen antes y después de la visita están relacionados con el esfuerzo dedicado a la búsqueda de información, el grado de incertidumbre, el nivel de implicación con el viaje, el número de fuentes secundarias de información consultadas y la duración e intensidad de la visita.

Palabras clave: Marketing turístico, Imagen de un destino turístico, Cambio de imagen de un destino, Factores de cambio de imagen.

Recibido: 18 de marzo de 2016

Devuelto para su revisión: 30 de junio de 2016

Aceptado: 20 de septiembre de 2016

* Facultad de Economía, Empresa y Turismo. Universidad de Las Palmas de Gran Canaria. Campus Universitario Tafira. 35017 LAS PALMAS DE GRAN CANARIA (España).E-mail: suny.beerli@ulpgc.es, josefa. martin@ulpgc.es

** Nazzareno Consulting S.L.U. Calle El Pilar 5, Oficina 9. 38002 SANTA CRUZ DE TENERIFE (España). E-mail: patrizionazzareno@hotmail.com 


\title{
Factors that explain the change in the image of a tourist destination before and after a visit
}

\begin{abstract}
This paper aims to study and empirically analyze the factors that explains the change in image before and after the visit to a tourist destination. In the empirical study it used a representative sample of tourists from the island of Tenerife (Canary Islands), which is one of the main tourist destinations in Spain. The results show that the factors that best explain the change in image before and after the visit are related to the effort dedicated to search for information, the degree of uncertainty, the level of involvement with the trip, the number of secondary sources of information consulted and the duration and intensity of the visit.
\end{abstract}

Keywords: Tourism marketing, Destination image, Destination image change, Destination image change factors.

\section{INTRODUCCIÓN}

A pesar del interés que ha suscitado la imagen de los destinos turísticos en la investigación académica, la mayoría de los trabajos se han realizado en la fase de elección del viaje (Pike y Ryan, 2004; Hyde 2008). Tal y como señalan algunos autores, se han realizado pocos estudios sobre la evolución de la imagen en las diferentes fases del viaje: pre, durante y después de la visita (Kim, Mckercher y Lee, 2009; Yilmaz et al., 2009). Estos estudios han demostrado que la imagen de un destino turístico puede cambiar de forma más o menos significativa, no sólo durante el viaje sino también después de la experiencia del viaje, con efectos sobre el nivel de satisfacción del turista y la probabilidad de recomendar el destino o de repetir la visita (Kim, Mckercher y Lee, 2009; Yilmaz et al., 2009). Profundizar en esta línea de investigación relativa a la evolución en el tiempo de la imagen de un destino en el desarrollo del proceso vacacional tiene importantes repercusiones para el sector. Los operadores del mercado y los responsables de marketing podrían ser capaces de construir una identidad más cercana a la realidad para aumentar la satisfacción y aprovechar aquellos elementos de la imagen que han demostrado, durante y después del viaje, ser los más aptos para sufrir modificaciones que impacten positivamente en el nivel de satisfacción (Bigné, Sánchez y Sanz, 2009).

Hemos de señalar también que hay muy pocos trabajos que hayan desarrollado modelos explicativos de las relaciones que existen entre la imagen de un destino turístico y la multitud de factores que la conforman (Beerli y Martín, 2004a, 2004b; Ryan y Cave, 2005; Tasci, 2007; Tasci y Gartner, 2007; Ferreira, 2011). Más concretamente, estos estudios han analizado el impacto de las fuentes de información primarias y secundarias y de las características personales del turista sobre el proceso de formación de la imagen del destino turístico. Sin embargo, no se encuentran trabajos que hayan evaluado cómo estos factores influyen sobre el cambio de imagen del destino turístico antes y después del viaje.

Sobre la base de lo anteriormente expuesto, con esta investigación se pretende contribuir a mejorar el conocimiento sobre los antecedentes del cambio que se produce en 
la imagen de un destino antes y después de su visita. Más concretamente, el objetivo general de esta investigación se centra en desarrollar y validar empíricamente un modelo explicativo que determine cuáles son los factores que influyen en el cambio de imagen pre y post-visita a través de un estudio empírico en el que se analiza el gap de la imagen en función de la información que tenían los turistas antes de realizar el viaje y al finalizar su estancia en el destino turístico.

\section{REVISIÓN DE LA LITERATURA}

El proceso de búsqueda de la información representa el momento inicial de formación o modificación de la imagen de un destino turístico y es un factor clave en todo el proceso de toma de decisión de la realización de un viaje a un destino turístico, ya que representa la fase inicial de activación del comportamiento del turista que intenta disminuir su percepción del riesgo de compra de un producto intangible (Gursoy y McCleary, 2004).

Con respecto al concepto de imagen, existe un acuerdo generalizado en la literatura de marketing turístico (Gartner, 1993; Baloglu y Brinberg, 1997; Walmsley y Young, 1998; Baloglu y McCleary, 1999a, 1999b; Baloglu y Mangaloglu, 2001; Chen y Uysal, 2002; Beerli y Martín, 2004a, 2004b; Pike y Ryan, 2004; Hosany, Ekinci y Uysal, 2006; Pike, 2009) en considerar la imagen como el resultado de tres componentes que están estrechamente interrelacionados: (1) perceptual/cognitivo, que está relacionado con las creencias de los individuos sobre los atributos que caracterizan a un lugar turístico; (2) afectivo, que se refiere a la respuesta emocional o sentimientos que los individuos expresan del lugar y (3) global, que se corresponde con la impresión global, positiva o negativa del lugar. Además, el componente cognitivo es un antecedente del afectivo, ya que las emociones están influenciadas también por elementos racionales y el componente cognitivo influye directamente sobre la imagen global de un destino e indirectamente a través la contribución del componente afectivo (Holbrook, 1978; Russel y Pratt, 1980; Anand, Holbrook y Stephens, 1988; Stern y Krakover, 1993; Beerli y Martín, 2004a, 2004b).

Centrándonos en los factores que influyen en el cambio de imagen pre y post-visita, la búsqueda de información del turista en términos de tiempo invertido en la búsqueda de información secundaria y número de fuentes de información secundaria consultadas influyen en la creación o modificación de la imagen de un destino por parte de un turista (Schmidt y Spreng, 1996; Fodness y Murray 1997, 1999; Gursoy y McCleary, 2004). La amplitud de las fuentes consultadas por el turista es una variable que influye en la formación de la imagen percibida del destino antes de su visita (Baloglu y McCleary, 1999a, 1999b), y es fundamental conocer qué factores determinan dicha amplitud y, por tanto, el esfuerzo que realiza el turista en la búsqueda de información antes de visitar el destino.

En primer lugar, el tiempo dedicado a la búsqueda de información influye en la cantidad de fuentes que puedan consultarse (Gursoy y McCleary, 2004). De hecho, el tiempo dedicado a la búsqueda de información secundaria externa deriva de la disponibilidad de tiempo y de la presión que la falta de tiempo ejerce sobre el turista. De esta forma, el valor percibido por los turistas bajo presión, debido a la inminente salida al destino, es mayor que el valor atribuido por los turistas que no se encuentran en esta situación. Así mismo, la búsqueda de información secundaria externa será mayor cuanto mayor sea la 
disponibilidad de tiempo (Beatty y Smith, 1987; Schmidt y Spreng, 1996). Por lo tanto, cuanto mayor sea el tiempo que dedican los turistas a recabar información, mayor será la cantidad de información recabada, conformándose los turistas una imagen más completa y exhaustiva antes de la visita (Balouglu y McCleary, 1999a, 1999b).

En segundo lugar, la necesidad de buscar información antes de realizar una compra es un comportamiento típico de los consumidores, los cuales a través de esta actividad reducen la incertidumbre y el riesgo inherente a la compra (Kotler et al., 1999). Debido a su naturaleza intangible, la compra de un viaje tiene un alto nivel de incertidumbre y el turista se activa para buscar información externa (Swarbrooke y Horner, 1999). Como ponen de manifiesto Gitelson y Crompton (1983), el individuo busca información sobre el destino porque (1) es un producto cuya compra es de alto riesgo y (2) es un producto que no se puede observar directamente antes de la compra por su naturaleza intangible. En general, un viaje presupone un desembolso considerable de dinero y una gran implicación de los turistas en la compra (Bonn et al., 1998); por ello, el proceso de búsqueda de información es considerado como un momento crítico para reducir la incertidumbre inherente a la compra (Quintal, Lee y Soutar, 2010).

En el proceso de búsqueda de la información, el reconocimiento de la necesidad es el primer paso por parte del turista (Moutinho, 1987) y surge a partir de las experiencias previas y de los recuerdos acumulados por el turista. Estos recursos internos son elementos del denominado conocimiento previo (Kersetter y Cho, 2004). Las personas que no están satisfechas con su nivel de conocimientos previos y reconocen que deben enfrentarse a una decisión caracterizada por la incertidumbre, tomarán las medidas necesarias para obtener información adicional (Bettman, 1979; Crotts, 1999; Quintal, Lee, Soutar, 2010).

Quintal et al. (2010) analizaron la influencia del riesgo (que se produce cuando la probabilidad de los resultados se conoce) y de la incertidumbre (que existe cuando no se conoce la probabilidad de los resultados) sobre el esfuerzo dedicado a la búsqueda de información. Los autores han demostrado que, durante la búsqueda de información sobre un destino turístico, el turista potencial desconoce la probabilidad de los resultados de su búsqueda y, por lo tanto, lo único que percibe es la incertidumbre. En tal sentido, la amplitud de la búsqueda de información varía de acuerdo con el grado de incertidumbre advertido y no tanto del nivel de riesgo. La variable riesgo afecta en un momento posterior, o sea, en la fase de evaluación de las alternativas, es decir, cuando el turista tiene la información necesaria sobre el destino para poder imaginar los posibles escenarios de su visita. Por lo tanto, cada vez que el visitante reconoce la necesidad de viajar y advierte la incertidumbre inherente a la compra, se esforzará para consultar todas las fuentes de información disponibles.

Por otra parte, Gursoy y Gavcar (2003) señalan que, debido a que el viaje es un producto caracterizado por su intangibilidad e incertidumbre, el turista durante su consumo muestra, en general, una mayor implicación que con respecto a cualquier otro tipo de producto. La implicación del turista en el viaje ha sido objeto de investigación por parte de la literatura, ya que explica en buena medida su comportamiento en la fase de búsqueda de información, durante la visita al destino y su intención futura de repetir. A este respecto, es posible identificar en la literatura posibles consecuencias que se derivan directamente de la implicación, como son una mayor intensidad motivacional, una 
mayor familiaridad con el destino, una reducción del riesgo percibido, la modificación de la imagen del destino, la capacidad de absorción de nueva información y la construcción de una relación de afiliación con el lugar (Mittal,1989; Ratchford y Vaughn,1989; Halloway y Robinson, 1995; Ryan 1995; Fodness y Murray, 1997; Vogt y Andereck, 2003; Pearce y Kang, 2009). Con respecto a la influencia de la implicación sobre la imagen del destino turístico, Martín et al. (2008) han demostrado que turistas con un nivel de implicación psicológica alto (muy motivado a viajar), pero con un bajo nivel de implicación situacional, o sea, con el lugar de visita, siguen teniendo una imagen positiva del destino y están dispuestos a recomendar el destino a otras personas, pero no a repetir el viaje. Mientras que los turistas con un alto nivel de implicación, tanto psicológica como situacional, tendrán una imagen positiva del destino, estarán dispuestos a recomendar el destino a otras personas y a repetir la experiencia. Por su parte, Gursoy y McCleary (2004) señalan que un individuo con un alto nivel de implicación estará más dispuesto a consultar una amplia variedad de fuentes de información, a aceptar pocas alternativas, a dar mucha importancia a la información, a procesar con mayor detalle la información recabada y a evaluar también los productos relacionados con el destino elegido. Como sugieren los autores, cuanto mayor es el nivel de implicación del turista tanto mayor será la probabilidad de que utilice un abanico más amplio de fuentes de información, dedique también más tiempo a buscar información y, por consiguiente, influya en el cambio de la imagen cognitiva pre y post-visita.

Sobre la base de lo expuesto en el presente epígrafe, se plantean las siguientes hipótesis relativas al esfuerzo dedicado a la búsqueda de información por parte de los turistas antes de visitar el destino:

H1: Cuanto mayor sea el tiempo dedicado a buscar información mayor será la amplitud o número de fuentes de información secundarias utilizadas.

H2: Cuanto más alto sea el nivel de incertidumbre percibido por el turista mayor será la amplitud o número de fuentes de información secundarias utilizadas.

H3: Cuanto mayor sea el nivel de implicación del individuo con el viaje mayor será el tiempo dedicado a buscar información.

H4: Cuanto más alto sea el nivel de incertidumbre percibido por el turista mayor será el tiempo dedicado a buscar información.

H5: Cuanto mayor sea el nivel de implicación del individuo con el viaje mayor será la amplitud o número de fuentes de información secundarias utilizadas.

H6: La amplitud o número de fuentes de información secundarias utilizadas influye directamente en el cambio de imagen cognitiva pre y post-visita.

H7: El nivel de implicación del individuo con el viaje influye en el cambio de imagen cognitiva pre y post-visita.

Otro tipo de antecedente de la imagen de un destino está relacionado con las características del viaje. En este sentido, en la literatura sobre imagen de destinos turísticos existen diferentes trabajos que han analizado cómo influyen las características del viaje en la imagen del destino, los cuales han estudiado, entre otras variables, la duración del viaje, la finalidad del viaje y la intensidad de la visita (Giltelson y Crompton, 1983; 
Fodness y Murray, 1997, 1999; Hyde y Lawson, 2003; Beerli y Martín, 2004a, 2004b; Hyde, 2008). A grandes rasgos, se establece que (1) la planificación de un viaje corto respecto a uno largo supone una conducta diferente del turista en la fase de búsqueda de información, que puede tener efectos sobre la percepción de la imagen del destino; (2) viajar por placer o viajar por negocios comporta una actitud diferente del turista, tanto en el momento preliminar al viaje como durante la visita, de ahí que se pueda afirmar que la imagen del destino se pueda ver afectada; y, finalmente, (3) el nivel de intensidad de la visita por parte del turista puede tener efectos sobre la imagen del lugar provocando cambios en la percepción inicial. Estas características del viaje influyen en dos fases diferentes del proceso de viaje: antes de la visita y durante la visita al destino. Cuando se hace referencia a la fase antes de la visita, se suele analizar la influencia de dos de estas variables en el proceso de búsqueda de información, esto es, la duración y la finalidad de la visita (Hyde y Lawson, 2003; Hyde, 2008); mientras que cuando se analiza la influencia de las características del viaje durante la visita, el análisis se ciñe más a cómo repercute la intensidad de la visita en la imagen de un destino (Beerli y Martín, 2004a, 2004b).

Hyde y Lawson (2003) ponen de manifiesto que existe una relación entre la duración del viaje y la planificación del mismo en la medida en que cuanto mayor sea la duración del viaje menores serán los esfuerzos dedicados a la planificación del viaje. En particular, los turistas interesados en viajes cortos tienden a maximizar el tiempo disponible y, por lo tanto, a planificar su viaje con detalle utilizando la información proporcionada por varias fuentes. Por el contrario, el turista que tiene disponible un período prolongado de viaje prefiere adoptar una programación flexible recurriendo a la consulta de un número menor de fuentes de información. Este resultado demuestra que la duración del viaje influye en el proceso de búsqueda de información en la medida en que afecta a la amplitud de las fuentes consultadas. De hecho, Hyde y Lawson (2003) señalan que, para viajes cortos, el turista tenderá a consultar muchas fuentes de información para optimizar su permanencia en el destino y, por tanto, desde nuestro punto de vista, influirá indirectamente en el cambio de imagen pre y post-visita. En esta línea, Tasci y Gartner (2007) ponen de manifiesto que la duración del viaje es uno de los componentes que afectan a la imagen de un destino turístico antes y durante el viaje.

Por otra parte, cuando un individuo efectúa la visita y vive la experiencia del viaje, la imagen se convierte en una imagen más realista, compleja y diferenciada (Phelps, 1986; Gartner y Hunt, 1987). La experiencia con el lugar reduce los estereotipos y conduce a un cambio de imagen, transformando lo que podríamos considerar como el paso de simples percepciones "en blanco y negro" a percepciones más definidas y completas (Beerli y Martín, 2004a, 2004b). Obviamente, esta evolución puede llevar a tres situaciones: la imagen se hace más simple, la imagen se hace más compleja y la imagen es la misma que se tenía antes de la visita (Fakeye y Crompton, 1991). Esta experiencia con el lugar se presenta como uno de los principales factores que impactan sobre la imagen durante y después del viaje y se concreta básicamente en la intensidad de la visita o nivel de interacción que ha tenido el individuo con el lugar. En los trabajos de Beerli y Martín (2004a, 2004b), la intensidad de la visita se asocia con el número de lugares de interés visitados. En cambio, otros autores han relacionado la intensidad de la visita con la experiencia directa con el 
destino o sea con el tiempo y las energías gastadas en la participación activa, comportamental y emotiva con el destino (Fesenmaier y Johnson 1989; Gursoy y Gavcar, 2003; Letho et al., 2004; Martín et al., 2008).

Resulta lógico admitir que la intensidad de la visita puede modificar la imagen percibida, ya que el nivel de interacción con el lugar determina una nueva percepción de la imagen más o menos lejana de la inicial. Partiendo de estas consideraciones, en este trabajo se plantea que la intensidad de la visita puede influir de forma directa en el cambio de imagen pre y post-visita en sus tres dimensiones, ya que si un turista no interactúa con el destino esta relación no se verá afectada, no ocurriendo lo mismo con un turista que interactúa mucho con el destino.

Sobre la base de lo expuesto, se plantean las siguientes hipótesis relacionadas con las características del viaje:

H8: La duración del viaje influye directamente en la amplitud de las fuentes de información consultadas, siendo menor el número de fuentes consultadas a medida que aumenta la duración del viaje, e indirectamente en el cambio de imagen cognitiva pre y post-visita.

H9a: La intensidad de la visita influye de forma directa en el cambio de imagen cognitiva pre y post-visita.

H9b: La intensidad de la visita influye de forma directa en el cambio de imagen afectiva pre y post-visita.

H9c: La intensidad de la visita influye de forma directa en el cambio de imagen global pre y post-visita.

\section{METODOLOGÍA}

La población objeto de estudio en la presente investigación se centra en los turistas de ocio de Tenerife (Islas Canarias), siendo la unidad de análisis el turista de ambos sexos, con 16 o más años de edad y que visita la isla de Tenerife por primera vez, procedente del extranjero y del resto de España.

La elección de la muestra se realizó en los lugares de interés turístico de Tenerife muy frecuentados por viajeros. Se siguió un método de selección empírico mediante las cuotas relativas a las dimensiones nacionalidad, sexo y edad, con afijación proporcional al número de turistas en cada una de las dimensiones establecidas. De esta forma se intentó garantizar la representatividad de la muestra, manteniendo la misma proporción que en la población para las distintas categorías de dichas dimensiones.

Se utilizaron encuestas personales a través de un cuestionario estructurado, que los turistas respondieron al final de su estancia en Tenerife, asegurándonos, de esta forma, que la experiencia del turista era reciente y completa. El tamaño muestral ascendió a 411 turistas, siendo, por tanto, el error asumido de un $\pm 4.93 \%$ para un intervalo de confianza del $95 \%$. El trabajo de campo se realizó en los meses de junio y julio de 2015. El perfil de los encuestados fue bastante similar al de la población en lo que se refiere a la distribución porcentual de la nacionalidad, género y edad. En la Tabla 1 se detalla el perfil sociodemográfico de la muestra. 


\section{Tabla 1 \\ PERFIL SOCIODEMOGRÁFICO \\ DE LA MUESTRA DE ENCUESTADOS}

\begin{tabular}{|c|c|c|}
\hline Características & $\mathbf{N}$ & $\%$ \\
\hline \multicolumn{3}{|l|}{ Género: } \\
\hline Hombre & 199 & 48.4 \\
\hline Mujer & 212 & 51.6 \\
\hline \multicolumn{3}{|l|}{ Edad: } \\
\hline 16-24 años & 32 & 7.8 \\
\hline 25-34 años & 106 & 25.8 \\
\hline 35-44 años & 62 & 15.1 \\
\hline 45-54 años & 80 & 19.5 \\
\hline 55-64 años & 81 & 19.7 \\
\hline Más de 64 años & 50 & 12.2 \\
\hline \multicolumn{3}{|l|}{ Nivel de estudios: } \\
\hline Sin estudios & 8 & 1.9 \\
\hline Primaria o ESO (ESO, FP1, EGB) & 42 & 10.2 \\
\hline Bachiller o FP2 & 196 & 47.7 \\
\hline Universitarios medios & 127 & 30.9 \\
\hline Universitarios superiores & 37 & 9.0 \\
\hline \multicolumn{3}{|l|}{ Clase social: } \\
\hline Alta & 38 & 9.2 \\
\hline Media-Alta & 78 & 19.0 \\
\hline Media-Media & 135 & 32.8 \\
\hline Media-Baja & 98 & 23.8 \\
\hline Baja & 4 & 1.0 \\
\hline \multicolumn{3}{|l|}{ Nacionalidad: } \\
\hline Alemania & 54 & 13.1 \\
\hline Reino Unido e Irlanda del Norte & 135 & 32.8 \\
\hline España (Península y Baleares) & 65 & 15.8 \\
\hline Países Nórdicos & 52 & 12.7 \\
\hline Otros países & 105 & 25.5 \\
\hline
\end{tabular}

Con respecto a las escalas de medida, se ha utilizado una escala Likert de 1 ítem y 7 puntos para medir el tiempo dedicado a buscar información (nada de tiempo, mucho tiempo), el nivel de incertidumbre antes del viaje a Tenerife (ninguna incertidumbre, muchísima incertidumbre) y la implicación con el destino (ningún interés por viajar a Tenerife, muchísimo interés por viajar a Tenerife). Para medir el gap de la imagen 
cognitiva, afectiva y global nos hemos fundamentado en los trabajos de Beerli y Martín (2004a y 2004b) y hemos utilizado una escala tipo Likert de 7 puntos y 15 ítems para medir la imagen cognitiva, de 2 ítems para medir la imagen afectiva (lugar agradable y lugar divertido o emocionante) y de 1 ítem para medir la imagen global. Para medir el cambio de imagen pre y post-visita, el encuestado valoró el grado en que cada ítem de la imagen cognitiva, afectiva y global había sido mejor, igual o peor de lo que esperaba de acuerdo con la información que tenía antes de realizar el viaje en una escala que iba desde -3 hasta +3 , donde -3 era mucho peor de lo esperado, 0 era igual a lo esperado y +3 era mucho mejor de lo esperado. Para medir la intensidad de la visita, siguiendo a Gitelson y Crompton (1983), Fodness y Murray (1997, 1999), Hyde y Lawson (2003), Beerli y Martín (2004a, 2004b) y Hyde (2008), hemos utilizado la interacción del turista con el destino a través de una escala dicotómica que recoge 18 lugares de interés turístico de Tenerife que pueden ser visitados durante la estancia en el destino por parte del turista. De esta forma la variable intensidad tomaba valores de 0 a 18. La duración del viaje se midió por el número de noches que ha estado el turista en Tenerife, que fue recodificada a 1 semana, 2 semanas o más de dos semanas. Finalmente, el número de fuentes de información consultadas se midió a través de escalas dicotómicas sobre el uso de 14 fuentes.

\section{RESULTADOS}

\subsection{Análisis y validación de las escalas de medida}

Como paso previo a la validación de la escala del cambio de imagen pre y post-visita, se realizó un análisis de frecuencia de los ítems que conformaban esta escala al objeto de analizar su distribución. Todos los ítems fueron valorados en una escala de $-3 \mathrm{a}+3$, donde -3 era mucho peor de lo esperado, 0 era igual a lo esperado y +3 mucho mejor de lo esperado. Los resultados reflejan que el porcentaje de gap negativo ( -3 a -1$)$ es muy reducido en todos los atributos del destino (inferior al 5\%). Debido a ello, hemos considerado más oportuno recodificar en un solo estado las categorías de $-3,-2,-1$ y 0 , que ha sido etiquetado como "Igual a lo esperado o ligeramente peor".

Los resultados finales del análisis factorial confirmatorio de segundo orden aplicado a la escala recodificada a cuatro estados del gap de la imagen cognitiva del destino arrojan la existencia de cuatro dimensiones, que han sido etiquetadas como "Recursos Naturales" (RECNAT), "Patrimonio e Infraestructuras" (PATRINF), “Actividades Turísticas" (ACTIVTUR) y "Entorno" (ENTORNO). Para analizar la validez discriminante de esta escala se estimó un nuevo modelo en el que todos los ítems se vinculan a un único factor. Los resultados de este nuevo modelo $\left[\chi^{2}(54)=295.12, p=0.000, \mathrm{CFI}=0.82, \mathrm{RMSEA}=0.10\right]$ evidencian la idoneidad de un modelo multidimensional $\left[\chi^{2}(50)=195.87, p=0.000, \mathrm{CFI}=0.89\right.$, $\mathrm{RMSEA}=0.08]$, ya que presenta mejores niveles de significación e índices de ajuste. De hecho, el análisis de diferencias de chi-cuadrado arroja la existencia de diferencias significativas (Dif. $\chi^{2}=99.25$, Dif. g.d.1. $=4, p=0.000$ ). Por tanto, esta escala de atributos del destino ha quedado formada finalmente por tres ítems en cada una de las dimensiones anteriormente señaladas y que se muestran en la Tabla 2. 
Tabla 2

ÍTEMS DEFINITIVOS DE LA ESCALA DE ATRIBUTOS DEL DESTINO

\begin{tabular}{|c|c|c|}
\hline Dimensiones & $\begin{array}{l}\text { Código } \\
\text { Ítem }\end{array}$ & Ítems \\
\hline \multirow{3}{*}{$\begin{array}{l}\text { Recursos } \\
\text { Naturales }\end{array}$} & V37R & Clima \\
\hline & V38R & Playas \\
\hline & V39R & $\begin{array}{l}\text { Recursos naturales (paisaje, parques naturales, flora y } \\
\text { fauna...) }\end{array}$ \\
\hline \multirow{3}{*}{$\begin{array}{l}\text { Patrimonio e } \\
\text { Infraestructuras }\end{array}$} & V41R & $\begin{array}{l}\text { Patrimonio cultural (monumentos históricos, museos, } \\
\text { gastronomía, conciertos, festivales...) }\end{array}$ \\
\hline & V42R & $\begin{array}{l}\text { Infraestructuras turísticas (alojamiento, restaurantes, } \\
\text { comercios, campos de golf, etc) }\end{array}$ \\
\hline & V43R & $\begin{array}{l}\text { Infraestructuras en general (carreteras, aeropuerto, } \\
\text { transporte, sanidad, internet...) }\end{array}$ \\
\hline \multirow{3}{*}{$\begin{array}{l}\text { Actividades } \\
\text { Turísticas }\end{array}$} & V44R & $\begin{array}{l}\text { Actividades de ocio turístico y recreo (parques } \\
\text { temáticos, turismo activo, golf, wellness...) }\end{array}$ \\
\hline & V45R & Ocio nocturno (bares, discotecas, pubs, casinos...) \\
\hline & V47R & $\begin{array}{l}\text { Actividades deportivas: vela, windsurf, cicloturismo, } \\
\text { deportes náutico }\end{array}$ \\
\hline \multirow{3}{*}{ Entorno } & V49R & Medioambiente (limpieza, aire puro...) \\
\hline & V50R & Seguridad \\
\hline & V51R & Relación calidad-precio \\
\hline
\end{tabular}

A pesar de que los resultados de este modelo de medida indican que es estadísticamente significativo $\left[\chi^{2}(50)=195.87, p=0.000\right]$, es preciso señalar que este estadístico depende del tamaño de la muestra analizada, de ahí la necesidad de analizar otros indicadores de ajuste. A este respecto, los resultados obtenidos muestran como otros indicadores de ajuste global del modelo se encuentran dentro de los valores recomendados por la literatura $(\mathrm{CFI}=0.89, \mathrm{NFI}=0.86, \mathrm{TLI}=0.85$, $\mathrm{RMSEA}=0.08)$, por lo que se puede concluir que el modelo especificado reproduce adecuadamente la matriz de covarianzas observada. Este modelo de medida muestra un ajuste adecuado, ya que el valor de CFI se sitúa entre 0.85 y 0.90 y el valor de RMSEA no supera el máximo recomendado de 0.08 (Mathieu y Taylor, 2006). Siguiendo a Anderson y Gerbing (1988) y como se observa en la Tabla 3, el modelo muestra una fiabilidad individual aceptable, ya que la relación existente entre cada ítem y su respectiva dimensión es estadísticamente significativa con pesos de regresión estandarizados superiores o muy cercanos en su mayoría a 0.7 y con valores del estadístico $t$ también significativos. En cuanto a las medidas de consistencia interna del constructo global del gap cognitivo, a diferencia de sus dimensiones, presenta unos niveles muy satisfactorios. Sin embargo, los valores del indicador de fiabilidad 
compuesta (FC) de sus dimensiones alcanzan valores cercanos a 0.70 y la mayoría de los de la varianza extraída (AVE) no superan el 0.50. Los valores del alfa de Cronbach corroboran los obtenidos en la fiabilidad compuesta. Estos resultados indican que el modelo de medida puede considerarse como válido, aunque sería recomendable también replicarlo en otros contextos e incluso ampliar o modificar el contenido de algunas de las dimensiones para elevar su nivel de fiabilidad. No obstante, puede afirmarse que la escala del gap de imagen cognitiva es un constructo de naturaleza multidimensional formado por cuatro dimensiones (véase Tabla 3).

Tabla 3

ANÁLISIS FACTORIAL CONFIRMATORIO DE LA ESCALA DEL GAP DE IMAGEN COGNITIVA DEL DESTINO

\begin{tabular}{|c|c|c|c|c|c|c|}
\hline \multicolumn{3}{|c|}{ Relaciones causales } & $\begin{array}{c}\text { Estimadores } \\
\text { estandarizados }\end{array}$ & $t$ & $p$ & $\begin{array}{c}\text { Consistencia } \\
\text { interna }\end{array}$ \\
\hline RECNAT & $\leftarrow$ & GAPIMAGCOG & 0.995 & & & \multirow{4}{*}{$\begin{array}{l}\mathrm{FC}=0.923 \\
\mathrm{AVE}=0.752 \\
\alpha=0.788\end{array}$} \\
\hline PATRINF & $\leftarrow$ & GAPIMAGCOG & 0.925 & 7.177 & 0.000 & \\
\hline ACTIVTUR & $\leftarrow$ & GAPIMAGCOG & 0.791 & 8.271 & 0.000 & \\
\hline ENTORNO & $\leftarrow$ & GAPIMAGCOG & 0.732 & 7.590 & 0.000 & \\
\hline V37R & $\leftarrow$ & RECNAT & 0.526 & & & \multirow{3}{*}{$\begin{array}{l}\mathrm{FC}=0.603 \\
\mathrm{AVE}=0.340 \\
\alpha=0.586\end{array}$} \\
\hline V38R & $\leftarrow$ & RECNAT & 0.521 & 7.781 & 0.000 & \\
\hline V39R & $\leftarrow$ & RECNAT & 0.688 & 9.141 & 0.000 & \\
\hline V41R & $\leftarrow$ & PATRINF & 0.517 & & & \multirow{3}{*}{$\begin{array}{l}\mathrm{FC}=0.665 \\
\mathrm{AVE}=0.402 \\
\alpha=0.670\end{array}$} \\
\hline V42R & $\leftarrow$ & PATRINF & 0.712 & 8.988 & 0.000 & \\
\hline V43R & $\leftarrow$ & PATRINF & 0.658 & 8.674 & 0.000 & \\
\hline V44R & $\leftarrow$ & ACTIVTUR & 0.753 & & & \multirow{3}{*}{$\begin{array}{l}\mathrm{FC}=0.703 \\
\mathrm{AVE}=0.443 \\
\alpha=0.709\end{array}$} \\
\hline $\mathrm{V} 45 \mathrm{R}$ & $\leftarrow$ & ACTIVTUR & 0.603 & 10.151 & 0.000 & \\
\hline V47R & $\leftarrow$ & ACTIVTUR & 0.631 & 10.508 & & \\
\hline V49R & $\leftarrow$ & ENTORNO & 0.692 & & 0.000 & \multirow{3}{*}{$\begin{array}{l}\mathrm{FC}=0.656 \\
\mathrm{AVE}=0.391 \\
\alpha=0.645\end{array}$} \\
\hline V50R & $\leftarrow$ & ENTORNO & 0.636 & 9.274 & & \\
\hline V51R & $\leftarrow$ & ENTORNO & 0.538 & 8.334 & 0.000 & \\
\hline
\end{tabular}

\subsection{Contraste de hipótesis}

Para contrastar las diferentes hipótesis y al objeto de conseguir una mayor parsimonia hemos utilizado como indicadores de la imagen cognitiva las cuatro variables creadas, una para cada una de las dimensiones del gap de la imagen cognitiva, que se han calculado como medias ponderadas de las puntuaciones dadas por los encuestados a los ítems que conforman cada dimensión ponderadas por los pesos de regresión de cada uno de ellos en los AFC. 
Para validar el modelo teórico propuesto sobre los antecedentes del gap entre la imagen pre y post-visita y contrastar las hipótesis de trabajo, se ha utilizado un modelo de ecuaciones estructurales a través del método de máxima verosimilitud. Los resultados indicaron que se trata de un modelo aceptable $\left[\chi^{2}(104)=336.91, p=0.000 ;\right.$ CFI $=0.85$; RMSEA=0.07], el valor de CFI está próximo a 0.90 y el de RMSEA por debajo de 0.08 (Mathieu y Taylor, 2006). Por otra parte, el porcentaje de varianza explicada del gap de la imagen global es relativamente elevado, situándose en un $60.9 \%$. En lo que respecta al gap de la imagen cognitiva y afectiva, el porcentaje de varianza explicada se eleva al $69.3 \%$ para la imagen afectiva, mientras que para la cognitiva se reduce al $15.2 \%$, lo cual es consecuencia de que existen otros factores adicionales no incluidos en el modelo que también contribuyen a explicar la imagen cognitiva, como, por ejemplo, la tipología de fuentes de información secundarias utilizadas. Finalmente, el porcentaje de varianza que se explica en el número de fuentes de información secundarias utilizadas es del $22.8 \%$. Como se muestra en la Figura 1, estos resultados demuestran que:

- El nivel de incertidumbre antes de la organización del viaje y el interés que despierta el destino en el turista antes de viajar influyen directa y positivamente en el número de fuentes de información secundaria utilizadas por el turista, no ocurriendo lo mismo con el tiempo que el turista dedique a la búsqueda de información $(~ \beta=0.183, p=0.010 ; \beta=0.373, p=0.000$, y $\beta=0.075, p=0.262$, respectivamente). Por tanto, se aceptan las hipótesis $\mathrm{H} 2$ y $\mathrm{H} 5$ y se rechaza $\mathrm{H} 1$. La no influencia del tiempo dedicado a la búsqueda en el número de fuentes puede ser una consecuencia de la mayor utilización de internet como fuente de información secundaria y, en ocasiones, la única, en cuyo medio los turistas suelen dedicar mucho tiempo. Por otra parte, también pueden existir otros factores, por ejemplo, de índole personal, que pueden afectar al número de fuentes y que no han sido incluidos en el modelo propuesto.

- Cuanto mayores sean los niveles de incertidumbre que posea un turista antes de organizar el viaje y de implicación o interés en el destino mayor será el tiempo que el turista dedique a la búsqueda de información $(\beta=0.320, p=0.000$ y $\beta=0.153$, $p=0.030$, respectivamente). Por tanto, se aceptan las hipótesis H3 y H4.

- La amplitud de las fuentes de información secundaria utilizadas por el turista influye directa y positivamente en el gap de la imagen cognitiva pre y post-visita, no ocurriendo lo mismo con el nivel de implicación del mismo con el viaje ( $\beta=0.231, p=0.003$ y $\beta=0.078, p=0.350$, respectivamente). Estos resultados dan apoyo empírico a la hipótesis H6, pero no a la H7. La no aceptación de H7 puede ser consecuencia del fuerte papel mediador ejercido por el número de fuentes de información consultadas entre el nivel de implicación del mismo con el viaje y el gap de la imagen cognitiva.

- Cuanto mayor es la duración del viaje mayor será el número de fuentes de información secundaria consultadas $(\beta=0.210, p=0.001)$, por lo que debemos rechazar la hipótesis $\mathrm{H} 8$, ya que el sentido de la relación es contrario al que se formuló teóricamente. Este resultado inesperado puede ser consecuencia de que el mayor porcentaje de los encuestados ha viajado de forma organizada y entre 8 y 14 
días de estancia, lo cual puede influir en que sean los turistas que viajan por su cuenta los que busquen más información cuando la duración del viaje es mayor.

- Cuanto mayor es el número de lugares de interés visitados por el turista, como un indicador de la intensidad de la visita, mayor es el gap de la imagen cognitiva $(\beta=0.232, p=0.003)$ y menor el gap de la imagen afectiva $(\beta=-0.114, p=0.080)$, no estando afectado el gap de la imagen global $(\beta=-0.149, p=0.429)$. Por consiguiente, se aceptan las hipótesis H9a y H9b y se rechaza la hipótesis H9c. El efecto mediador que ejerce el gap de la imagen cognitiva y afectiva entre la intensidad de la visita y el gap de la imagen global puede ser el motivo que justifique el rechazo de la hipótesis $\mathrm{H} 9 \mathrm{c}$.

Para una mayor comprensión, en la Tabla 4 se recoge un resumen de las hipótesis que han sido aceptadas y rechazadas.

\section{Figura 1 \\ RESULTADOS DEL MODELO DE ANTECEDENTES DEL GAP DE LA IMAGEN}

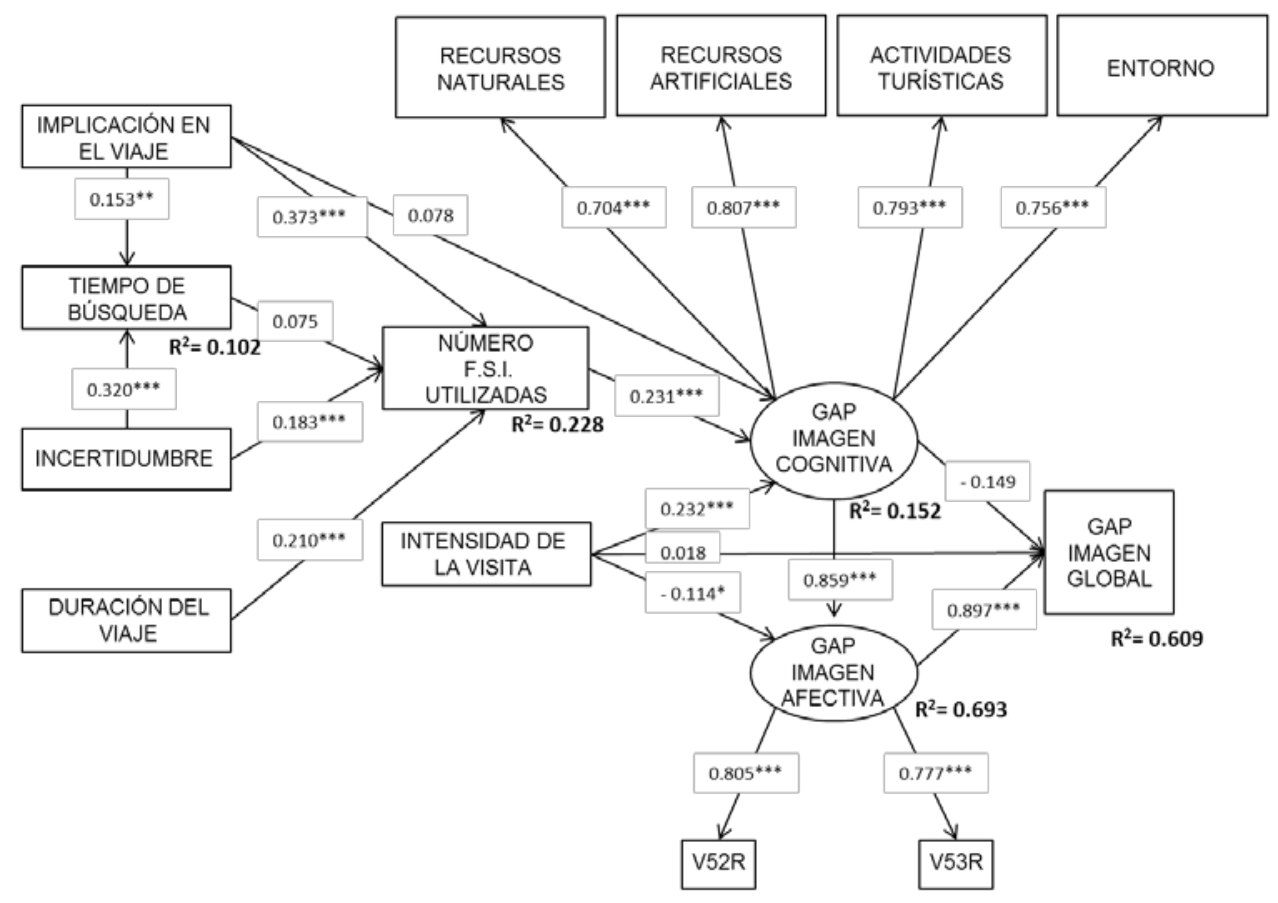

AJUSTE DEL MODELO

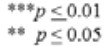

* $p \leq 0.1$

$X^{2}(154)=336.91(p=0.000), C M I N / D F=3.24$

$\mathrm{CFI}=0.85, \mathrm{NFI}=0.80, \mathrm{TLI}=0.77, \mathrm{RMSEA}=0.07$ 
Tabla 4

RESUMEN DEL CONTRASTE DE HIPÓTESIS

\begin{tabular}{|c|c|c|}
\hline & HIPÓTESIS & RESULTADO \\
\hline H1 & $\begin{array}{l}\text { Cuanto mayor sea el tiempo dedicado a buscar información } \\
\text { mayor será la amplitud o número de fuentes de información } \\
\text { secundarias utilizadas. }\end{array}$ & Rechazada \\
\hline H2 & $\begin{array}{l}\text { Cuanto más alto sea el nivel de incertidumbre percibido por } \\
\text { el turista mayor será la amplitud o número de fuentes de } \\
\text { información secundarias utilizadas. }\end{array}$ & Aceptada \\
\hline H3 & $\begin{array}{l}\text { Cuanto mayor sea el nivel de implicación del individuo con } \\
\text { el viaje mayor será el tiempo dedicado a buscar información. }\end{array}$ & Aceptada \\
\hline H4 & $\begin{array}{l}\text { Cuanto más alto sea el nivel de incertidumbre percibido } \\
\text { por el turista mayor será el tiempo dedicado a buscar } \\
\text { información. }\end{array}$ & Aceptada \\
\hline H5 & $\begin{array}{l}\text { Cuanto mayor sea el nivel de implicación del individuo } \\
\text { con el viaje mayor será la amplitud o número de fuentes de } \\
\text { información secundarias utilizadas. }\end{array}$ & Aceptada \\
\hline H6 & $\begin{array}{l}\text { La amplitud o número de fuentes de información secundarias } \\
\text { utilizadas influye directamente en el cambio de imagen } \\
\text { cognitiva pre y post-visita. }\end{array}$ & Aceptada \\
\hline H7 & $\begin{array}{l}\text { El nivel de implicación del individuo con el viaje influye en } \\
\text { el cambio de imagen cognitiva pre y post-visita. }\end{array}$ & Rechazada \\
\hline H8 & $\begin{array}{l}\text { La duración del viaje influye directamente en la amplitud } \\
\text { de las fuentes de información consultadas, siendo menor } \\
\text { el número de fuentes consultadas a medida que aumenta la } \\
\text { duración del viaje, e indirectamente en el cambio de imagen } \\
\text { cognitiva pre y post visita. }\end{array}$ & Rechazada \\
\hline H9a & $\begin{array}{l}\text { La intensidad de la visita influye de forma directa en el } \\
\text { cambio de imagen cognitiva pre y post-visita. }\end{array}$ & Aceptada \\
\hline H9b & $\begin{array}{l}\text { La intensidad de la visita influye de forma directa en el } \\
\text { cambio de imagen afectiva pre y post-visita. }\end{array}$ & Aceptada \\
\hline H9c & $\begin{array}{l}\text { La intensidad de la visita influye de forma directa en el } \\
\text { cambio de imagen global pre y post-visita. }\end{array}$ & Rechazada \\
\hline
\end{tabular}

\section{CONCLUSIONES E IMPLICACIONES}

Una de las principales aportaciones de esta investigación a la literatura científica sobre el cambio de la imagen de un destino turístico ha sido analizar los antecedentes o las causas que conducen al cambio de la imagen durante y después de la visita. La relevancia 
de este trabajo radica en la carencia de estudios en esta línea, ya que los escasos estudios académicos sobre este tópico del gap se han centrado en profundizar en las consecuencias que un cambio de imagen tiene en la satisfacción y lealtad del turista (Yilmaz et al., 2009; Kim, McKercher y Lee, 2009). Es por ello que esta investigación ha prestado una especial atención a las causas que originan el cambio de imagen, evidenciando cuáles son los factores que afectan al cambio de la imagen y que deben ser considerados y gestionados por los operadores del mercado turístico para que el destino pueda seguir manteniendo su éxito, ya que la imagen es uno de los factores más relevantes del mismo. A partir de la revisión teórica se ha planteado una serie de hipótesis que, de forma conjunta, dibujan un modelo teórico explicativo del cambio de imagen de un destino turístico, y que fueron contrastadas con la información recabada a partir de una encuesta personal realizada en una muestra significativa y representativa del perfil del turista que visita Tenerife. Por tanto, el diseño de este modelo constituye en sí mismo una primera contribución académica al campo de la investigación sobre el cambio de imagen de un destino turístico desde una perspectiva integradora y holística.

La contrastación de las hipótesis sobre la relación existente entre los antecedentes vinculados al esfuerzo dedicado a la búsqueda de información y el cambio de imagen, permite afirmar que el nivel de incertidumbre, el interés que despierta el destino en el turista antes de viajar y el número de fuentes de información secundarias utilizadas por el turista influyen de forma conjunta en el cambio de la imagen cognitiva, de los cuales tan sólo el número de fuentes es un antecedente directo del mismo. Con este resultado se confirma que la percepción de un riesgo mayor antes de viajar repercute en la búsqueda de una mayor cantidad de fuentes de información y en el tiempo dedicado a su búsqueda. Finalmente, se verifica también la relación directa y positiva entre el nivel de interés o implicación que tenga el turista antes de efectuar el viaje con la cantidad de fuentes de información consultadas y el tiempo dedicado a la búsqueda. Estos resultados ponen de manifiesto que (1) el tiempo es una variable del comportamiento del turista, que está condicionada tanto por la incertidumbre que despierta el destino como por el interés del turista en el viaje, y (2) la cantidad de fuentes de información consultadas por el turista influye en el cambio de la imagen cognitiva.

Por otra parte, los resultados de este trabajo de investigación ponen de manifiesto que cuanto mayor es la duración del viaje mayor será el número de fuentes de información consultadas, resultado que no concuerda con el esperado, por lo que no se ha podido verificar la hipótesis en la que se establecía que cuanto menor es la duración del viaje mayor es la amplitud de fuentes consultadas. Los motivos que pueden justificar este resultado inesperado pueden derivarse de la circunstancia de que los turistas que vienen a Tenerife por primera vez tienden a realizar un viaje relativamente largo, lo que les permite visitar ampliamente el destino y para ello recaban amplia información antes de visitarlo. Por otra parte, pueden existir otras variables que condicionen el comportamiento del turista antes de visitar el destino, como, por ejemplo, que organicen por su cuenta el viaje o lo hagan de forma programada con agencias de viaje.

En lo que a la intensidad de la visita se refiere, los resultados indican, en primer lugar, que cuanto mayor es el número de lugares de interés visitados por el turista o la intensidad de la visita, mayor es el cambio positivo de la imagen cognitiva. Es decir, los turistas que 
durante su estancia visitan un número más elevado de lugares de interés turístico tienen una imagen cognitiva del destino mucho mejor de la inicial. Probablemente, este resultado se debe al hecho de que muchos lugares atractivos del destino no son lo suficientemente conocidos por los turistas antes de la visita y sorprenden favorablemente a los visitantes. En segundo lugar, se observa que el efecto de la intensidad de la visita en el cambio de imagen afectiva es negativo, por lo que cuanto mayor es el número de lugares visitados menor resulta el gap de la imagen afectiva. Por tanto, los turistas que antes de viajar consideran que el destino es un lugar agradable, divertido o emocionante, cuanto más lugares visitan durante su estancia menos cambian su juicio sobre estos atributos de la imagen afectiva, por lo que el gap disminuye. Finalmente, los resultados evidencian también que no existe ninguna relación entre intensidad de la visita y el cambio de la imagen global del destino.

Con esta investigación se ha tratado de contribuir a un mayor conocimiento en el ámbito de la imagen de los destinos turísticos y, más concretamente, en el concepto de cambio de la imagen después de la visita al destino, tanto en lo que al ámbito académico se refiere como al profesional. Desde un punto de vista académico, se ha pretendido aportar un marco conceptual que permita seguir avanzando en el desarrollo del tópico de la imagen de los destinos turísticos para comprender mejor el proceso de evolución de la misma en las diferentes fases del viaje. En este sentido, se pretende dar respuesta a la necesidad que proclaman diversos autores de estudiar en mayor profundidad los factores que influyen en el cambio de la imagen percibida de los destinos turísticos, dadas las escasas evidencias empíricas existentes al respecto, y contribuir a cubrir la laguna existente en la literatura académica. A este respecto, y como ya se ha comentado anteriormente, con este modelo lo que se pretende es analizar de una forma integradora y holística los factores determinantes del cambio de imagen. Más concretamente, en primer lugar y partiendo de una estructura conceptual de la imagen con soporte teórico, se ha verificado que la imagen de los destinos turísticos puede estar sujeta a un cambio durante y después de la visita y que los factores responsables del cambio están (1) relacionados con el proceso de búsqueda de información secundaria y (2) vinculados a las características del viaje. En segundo lugar y tomando como referencia la literatura fragmentada existente, se ha diseñado y validado empíricamente un modelo con el que se pretende contribuir a un mayor conocimiento de los factores o antecedentes que determinan la formación del gap de la imagen de los destinos turísticos. Desde un punto de vista práctico, la comprensión global del proceso de formación del gap de la imagen de los destinos turísticos y de los factores que influyen en este cambio ayudarán a los operadores del sector y a las instituciones públicas responsables de la gestión comercial de los destinos a proyectar una imagen lo más cercana posible a la realidad. Sería recomendable que los operadores de mercado y las instituciones públicas responsables de la imagen de un destino turístico evalúen el potencial real en la comunicación del destino para que la imagen que se proyecta del destino sea similar a la que los turistas perciben después de visitar el destino. Por otra parte, el organismo responsable de la comercialización del destino debe potenciar la página web del destino y aportar información amplia, objetiva y real de los lugares de interés turístico del destino que motive a los turistas a tener una mayor interacción con el destino, ya que cuanta más información dispongan los turistas y mayor interacción tengan con el destino más positivo es el gap 
de la imagen cognitiva del destino. Centrándonos en el destino turístico de Tenerife, los resultados de la investigación empírica ponen de manifiesto que la imagen proyectada de Tenerife genera un cambio positivo de la imagen, ya que los turistas durante su estancia experimentan una realidad mucho mejor de la que esperaban.

Si bien hemos tratado de llevar a cabo un trabajo de investigación siguiendo criterios de rigurosidad científica con el objetivo de aportar una evidencia empírica que contribuya a un mayor conocimiento y comprensión del proceso de formación del gap de la imagen de los destinos turísticos, somos conscientes de sus limitaciones, tanto de orden conceptual como metodológico. Desde un punto de vista conceptual, hemos tratado de desarrollar y validar empíricamente los factores que influyen en el cambio de la imagen percibida de un destino turístico, aunque somos conscientes de que existen otros antecedentes que pueden afectar al cambio de imagen y que no han sido incluidos en la presente investigación debido a que este trabajo de investigación, como cualquier otro, se circunscribe al contexto de sus propios objetivos. Además, sería interesante ampliar el análisis a los turistas repetidores para averiguar el efecto del conocimiento previo y de la familiaridad sobre un eventual gap de la imagen. Desde una perspectiva metodológica, este trabajo, como cualquier otra investigación empírica, presenta limitaciones que afectan a la evaluación y generalización de sus resultados. En línea con el diseño transversal de la investigación, debemos interpretar con cautela las relaciones causales que se desprenden de nuestro estudio, ya que su diseño no permite afirmar de forma estricta el cumplimiento de las condiciones de causalidad, en tanto y en cuanto no podemos asegurar de forma estricta que los cambios en la causa implican cambios en el efecto. En los modelos estructurales la causalidad debe entenderse en términos de asociación estadística y no bajo las condiciones de un diseño experimental. No obstante, hemos tratado de plantear relaciones causales sustentadas teóricamente sobre la base de la fundamentación teórica planteada en el apartado de revisión de la literatura. Finalmente, la generalización de los resultados constituye otra limitación, ya que el ámbito de la investigación sólo permite generalizar los resultados de los análisis a la población de la que procede la muestra y al destino turístico de Tenerife, por lo que sería aconsejable replicar esta investigación en otros contextos y analizar los factores que influyen en el cambio de la imagen percibida en otros destinos turísticos.

\section{REFERENCIAS BIBLIOGRÁFICAS}

ANAND, P., HOLBROOK, M.B. y STEPHENS, D. (1988): «The formation of affective judgements: The Cognitive-Affective Model versus the independence hypothesis», Journal of Consumer Research, vol. 15, pp. 386-391.

ANDERSON, J.C. y GERBING, D.W. (1988): «Structural Equation Modeling in Practice: A Review and Recommended Two-Step Approach», Psychological Bulletin, vol. 103, pp. 411-423.

BALOGLU, S. y BRINBERG, D. (1997): «Affective images of tourism destinations», Journal of Travel Research, vol. 35, nº4, pp. 11-15.

BALOGLU, S. y MANGALOGLU, M. (2001): «Tourism Destination Images of Turkey, Egypt, Greece, and Italy as Perceived by US-based Tour Operators and Travel Agents», Tourism Management, vol. 22, $\mathrm{n}^{\circ}$ 1, pp. 1-9. 
BALOGLU, S. y MCCLEARY, K.W. (1999a): «A Model of Destination Image Formation», Annals of Tourism Research, vol. 26, n 4, pp. 868-897.

BALOGLU, S. y MCCLEARY, K.W. (1999b): «U.S. International Travellers’ Images of Four Mediterranean Destinations: A Comparison of Visitors and No visitors», Journal of Travel Research, vol. 38, ${ }^{\circ}$ 2, pp. 144-152.

BEATTY, S. y SMITH, S. (1987): «External Search Effort: An Investigation Across

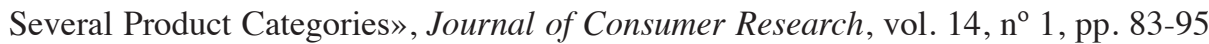

BEERLI, A. y MARTÍN, J.D. (2004a): «Factor influencing destination image», Annals of Tourism Research, vol. 31, n 3, pp. 657-681.

BEERLI, A. y MARTÍN, J.D. (2004b): «Tourists' characteristics and the perceived image of tourist destinations: A quantitative analysis - a case study of Lanzarote, Spain», Tourism Management, vol. 25, $\mathrm{n}^{\circ}$ 5, pp. 623-636.

BETTMAN, J.R. (1979): «Issues in Research on Consumer Choice», Advances in Consumer Research, vol. 6, pp. 214-217.

BIGNÉ, E., SÁNCHEZ, I. y SANZ, S. (2009): «The functional-psychological continuum in the cognitive image of a destination: A confirmatory analysis», Tourism Management, vol. 30, $\mathrm{n}^{\mathrm{o}} 5$, pp. 715-723.

BIGNÉ, E., SÁNCHEZ, M.I. y SÁNCHEZ, J. (2001): «Tourism Image, Evaluation Variables and after Purchase Behaviour: Inter-relationship», Tourism Management, vol. 22, $\mathrm{n}^{\circ}$ 6, pp. 607-616.

BONN, M.A., FURR, H.L. y SUSSKING, A.M. (1998): «Using the Internet as a pleasure travel planning tool. An examination of the sociodemographic and behavioural characteristics amongst internet users and nonusers», Journal of Hospitality and Tourism Research, vol. 22, n 3, pp. 303-317.

CHEN, J. y UYSAL, M. (2002): «Market Positioning Analysis: A Hybrid Approach», Annals of Tourism Research, vol. 29, nº4, pp. 987-1003.

CROTTS, J. (1999): «Consumer Decision Making and Pre-purchase Information Search», en A. Pizam y Y. Mansfeld (Eds.): Consumer Behavior in Travel and Tourism, pp. 149-168.

FAKEYE, P.C. y CROMPTON, J.L. (1991): «Image Differences Between Prospective, First-Time, and Repeat Visitors to the Lower Rio Grande Valley», Journal of Travel Research, vol. 30, $\mathrm{n}^{\mathrm{O}} 2$, pp. 10-16.

FERREIRA S. (2011): «Destination image: Origins, Developments and Implications», Pasos, Revista de Turismo y Patrimonio Cultural, vol. 9, n 2, pp. 305-315.

FESENMAIER, D.R. y JOHNSON, B. (1989): «Involvement Based Segmentation: Implications for Travel Marketing in Texas», Tourism Management, vol. 10, $\mathrm{n}^{\circ} 4$, pp. 293-300.

FODNESS, D. y MURRAY, B. (1997): «Tourist information search», Annals of Tourism Research, vol. 24, no 3, pp. 503-523.

FODNESS, D. y MURRAY, B. (1999): «A Model of Tourist Information Search Behavior», Journal of Travel Research, vol. 37, pp. 220-232.

GARTNER, W.C. (1993): «Image Formation Process», Journal of Travel and Tourism Marketing, vol. 2, no 2/3, pp. 191-215. 
GARTNER, W.C. y HUNT, J.D. (1987): «An Analysis of State Image Change over a Twelve-Year Period (1971-1983)», Journal of Travel Research, vol. 26, n 2, pp. 15-19. GITELSON, R.J. y CROMPTON, J.L. (1983): «The Planning Horizons and Sources of Information Used by Pleasured Vacationers», Journal of Travel Research, vol. 21, $\mathrm{n}^{\circ}$ 3, pp. 2-27.

GURSOY, D. y MCCLEARY, K.W. (2004): «An Integrative Model of Tourists' Information Search Behavior», Annals of Tourism Research, vol. 31, n 2, pp. 353-373.

GURSOY, D. y GAVCAR, E. (2003): «International leisure tourists' involvement profile», Annals of Tourism Research, vol. 30, n 4, pp. 906-926.

HOLBROOK, M.B. (1978): «Beyond attitude structure: toward the informational determinants of attitude», Journal of Marketing Research, vol. 15, no 4, pp. 545-556.

HOLLOWAY, J.C. y ROBINSON, C. (1995): «Marketing for Tourism» ( $3^{\mathrm{a}}$ ed.), United Kingdom, Adisson Wesley Longman.

HOSANY, S., EKINCI, Y. y UYSAL, M. (2006): «Destination image and destination personality: An application of branding theories to tourism places», Journal of Business Research, vol. 59, $\mathrm{n}^{\circ}$ 5, pp. 638-642.

HYDE, K. (2008): «Information Processing and Touring Planning Theory», Annals of Tourism Research, vol. 35, no 3, pp. 712-731.

HYDE, K. y LAWSON, R. (2003): «The nature of independent travel», Journal of Travel Research, vol. 42, $\mathrm{n}^{\circ}$ 1, pp. 13-23.

KERSTETTER, D. y CHO, M.H. (2004): «Prior Knowledge, credibility and information search», Annals of Tourism Research, vol. 31, n 4, pp. 961-985.

KIM, S., MCKERCHER, B. y LEE, H. (2009): «Tracking Tourism Destination Image Perception», Annals of Tourism Research, vol. 36, $\mathrm{n}^{\circ}$ 4, pp. 715-718.

KOTLER, P., ARMSTRONG, G., SAUNDRES, J. y WONG, V. (1999): «Principles of Marketing», Prentice Hall Europe.

LEHTO, X.Y., CAI, L.A., O'LEARY, J.T. y HUAN, T.C. (2004): «Tourist shopping preferences and expenditure behaviors: The case of the Taiwanese outbound market», Journal of Vacation Marketing, vol. 10, $\mathrm{n}^{\circ}$ 4, pp. 320-332.

MARTÍN, D., COSSIO, F. y MARTÍN, E. (2008): «The moderating effect of customer's involvement on the relationship between customer perceptions and its future behavior», Actas del Congreso Nacional de Marketing, Maspalomas (Islas Canarias).

MATHIEU, J.E. y TAYLOR, S.R. (2006): «Clarifying conditions and decision points for mediational type inferences in Organizational Behavior», Journal of Organizational Behavior, vol. 27, $\mathrm{n}^{\circ} 8$, pp. 1031-1056.

MITTAL, B. (1989): «A Theoretical Analysis of two Recent Measures of Involvement», Advances in Consumer Research, vol. 16, pp. 697-702.

MOUTINHO, L. (1987): «Consumer Behavior in Tourism», European Journal of Marketing, vol. 21, n ${ }^{\circ} 10$, pp. 5-44.

PEARCE, P.L. y KANG, M.H. (2009): «The effects of prior and recent experience on continuing interest in tourist settings», Annals of Tourism Research, vol. 36, $\mathrm{n}^{\circ} 2$, pp. 172-190.

PHELPS, A. (1986): «Holiday Destination Image - The Problem of Assessment: An Example Developed in Menorca», Tourism Management, vol. 7, n 3, pp. 168-180. 
PIKE, S. (2009): «Destination brand positions of a competitive set of near-home destinations», Tourism Management, vol. 30, nº 6, pp. 857-866.

PIKE, S. y RYAN, C. (2004): «Destination positioning analysis through a comparison of cognitive, affective and conative perceptions», Journal of Travel Research, vol. 42, $\mathrm{n}^{\mathrm{o}} 4$, pp. 333-342.

QUINTAL, V.A., LEE, J.A. y SOUTAR, G.N. (2010): «Tourists' Information Search: The Differential Impact of Risk and Uncertainty Avoidance», International Journal of Tourism Research, vol. 12, n 4, pp. 321-333.

RATCHFORD, B.T. y VAUGHN, R. (1989): «On the Relationship Between Motives and Purchase Decisions: Some Empirical Approaches», Advances in Consumer Research, vol. 16, pp. 293-299.

RUSSEL, J.A. y PRAT, G. (1980): «A description of affective quality attributed to environment», Journal of Personality and Social Psychology, vol. 38, n 2, pp. 311-322.

RYAN, C. (1995): «Researching Tourist Satisfaction», London, Routledge.

RYAN, C. y CAVE, J. (2005): «Structuring destination image: A qualitative approach», Journal of Travel Research, vol. 44, $\mathrm{n}^{\circ}$ 2, pp. 143-150.

SCHMIDT, J.B. y SPRENG, R.A. (1996): «A proposed model of external consumer information search», Journal of the Academy of Marketing Science, vol. 24, $\mathrm{n}^{\circ} 3$, pp. 246-256.

STERN, E. y KRAKOVER, S. (1993): «The Formation of a Composite Urban Image», Geographical Analysis, vol. 25, n 2, pp. 130-146.

SWARBROOKE, J. y HORNER, S. (1999): «Consumer Behavior in Tourism», Great Britain, Butterworth-Heinemann.

TASCI, A.D.A. (2007): "Assessment of factors influencing destination image using a multiple regression model», Tourism Review, vol. 62, n 2, pp. 23-30.

TASCI, A.D.A. y GARTNER, W.C. (2007): «Destination Image and Its Functional Relationships», Journal of Travel Research, vol. 45, n 4, pp. 413-425.

VOGT, C.A. y ANDERECK, K.L. (2003): «Destination Perceptions Across a Vacation», Journal of Travel Research, vol. 41, nº 4, pp. 348-354.

WALMSLEY, D.J. y YOUNG, Y.M. (1998): «Evaluative Images and Tourism: The Use of Personal Constructs to Describe the Structure of Destination Images», Journal of Travel Research, vol. 36, no 3, pp. 65-69.

YILMAZ, Y., YILMAZ, Y., IÇIGEN, E.T., EKIN Y. y UTKU, B.D. (2009): «Destination Image: A Comparative Study on Pre and Post Trip Image Variations», Journal of Hospitality Marketing \& Management, vol. 18, $\mathrm{n}^{\circ}$ 5, pp. 461-479. 